\title{
Reconstrução de Trânsito Intestinal: Caracterização sociodemográfica, clínica e terapêutica da clientela
}

\author{
Bowel Transit Reconstruction: Sociodemographic, clinical and therapeutic characterization of \\ clientele
}

Reconstrucción del Tránsito Intestinal: Caracterización sociodemográfica, clínica y terapéutica de

la clientela

Recebido: 20/04/2021 | Revisado: 01/05/2021 | Aceito: 06/05/2021 | Publicado: 20/05/2021

Tamires Souza Rodrigues

ORCID: https://orcid.org/0000-0002-5737-5618

União das Faculdades dos Grandes Lagos, Brasil

E-mail: tsr.albuquerque@gmail.com

Janderson Cleiton Aguiar

ORCID: https://orcid.org/0000-0002-6095-8689

Universidade de São Paulo, Brasil E-mail: jcaguiar@usp.br

Natalia Sperli Geraldes Marin dos Santos Sasaki

ORCID: https://orcid.org/0000-0002-8627-9713

União das Faculdades dos Grandes Lagos, Brasil E-mail: nsperli@gmail.com

Wagner Felipe dos Santos Neves ORCID: https://orcid.org/0000-0001-9989-7273

Universidade de São Paulo, Brasil

E-mail: wagnerfelipeneves@usp.br

André Aparecido da Silva Teles ORCID: https://orcid.org/0000-0002-0548-9592

Universidade de São Paulo, Brasil E-mail: andreteles@usp.br

Vanessa Damiana Menis Sasaki ORCID: https://orcid.org/0000-0001-9745-7573

Universidade de São Paulo, Brasil

E-mail: vanessasasaki@hotmail.com

Antonio Jorge Silva Correa Júnior ORCID: https://orcid.org/0000-0003-1665-1521

Universidade de São Paulo, Brasil

E-mail: antoniocorreajunior@usp.br

Camila Maria Silva Paraizo Horvath

ORCID: https://orcid.org/0000-0002-3574-7361

Universidade de São Paulo, Brasil

E-mail: camilaparaizo@usp.br

Tatiana Mara da Silva Russo

ORCID: https://orcid.org/0000-0002-8077-0595

Universidade de São Paulo, Brasil E-mail: tatiana.russo@usp.br

Helena Megumi Sonobe

ORCID: https://orcid.org/0000-0003-3722-0835

Universidade de São Paulo, Brasil

E-mail: megumi@eerp.usp.br

\begin{abstract}
Resumo
Objetivo: Analisar as características sociodemográficas, clínicas e terapêuticas de pessoas submetidas à Reconstrução de Trânsito Intestinal. Metodologia: trata-se de um estudo retrospectivo, descritivo-analítico, quantitativo, realizado em um centro de reabilitação especializado em uma cidade paulista, com levantamento de 134 prontuários de pacientes que realizaram Reconstrução de Trânsito Intestinal (RTI), aprovado pelo Comitê de Ética em Pesquisa (Parecer $\mathrm{n}^{\circ}$ 2.831.709). Resultados e discussão: Com a análise de prontuários da população de pacientes que realizaram RTI 134 (100\%), obteve-se média de idade de 57,3 anos, predominantemente do sexo masculino 78 (58\%), do atendimento pelo sistema público de saúde 122 (91\%); do diagnóstico oncológico 63 (47\%); de cirurgia de urgência 88 (66\%); e de 92 (69\%) pessoas com colostomias. Houve correlação estatisticamente significante entre tipo de estomia, caráter da cirurgia, técnica cirúrgica e o diagnóstico de indicação cirúrgica, diferentemente das variáveis cirurgia e o tempo de
\end{abstract}


estomia. Conclusão: Há necessidade de prevenção e diagnóstico precoce de CCR, além do planejamento da assistência à saúde para o seguimento de controle cirúrgico e oncológico, para agilização de reconstrução de trânsito intestinal (RTI) quando possível, além do preparo desta clientela para as mudanças na vida após RTI, bem como no gerenciamento da previsão e provisão de equipamentos coletores e adjuvantes, o que requer integração das ações dos diferentes níveis de atendimento desta clientela.

Palavras-chave: Estomas cirúrgicos; Assistência prestada ao paciente; Perfil de saúde.

\begin{abstract}
Objective: To analyze the sociodemographic, clinical and therapeutic characteristics of people undergoing bowel transit reconstruction. Methodology: this is a retrospective, descriptive-analytical, quantitative study, carried out in a specialized rehabilitation center in a city of São Paulo, with a survey of 134 records of patients who underwent Bowel Transit Reconstruction (BTR), approved by the Ethics Committee in Research ( $\left.\mathrm{n}^{\mathbf{o}} 2,831,709\right)$. Results and discussion: With the analysis of medical records of the population of patients who underwent BTR 134 (100\%), an average age of 57.3 years was obtained, predominantly male $78(58 \%)$, of the attendance by the public health system. health $122(91 \%)$; oncologic diagnosis 63 (47\%); urgent surgery 88 (66\%); and $92(69 \%)$ people with colostomies. There was a statistically significant correlation between type of ostomy, character of the surgery, surgical technique and the diagnosis of surgical indication, differently from the surgery variables and the time of ostomy. Conclusion: There is a need for prevention and early diagnosis of colorectal cancer, in addition to planning health care for the follow-up of surgical and oncological control, to speed up BTR when possible, in addition to preparing this clientele for changes in after BTR, as well as in the management of the forecast and provision of collection equipment and adjuvants, which requires integration of the actions of the different levels of service of this clientele.
\end{abstract}

Keywords: Surgical stomas; Patient care; Health profile.

\title{
Resumen
}

Objetivo: Analizar las características sociodemográficas, clínicas y terapéuticas de personas sometidas a reconstrucción del tránsito intestinal. Metodología: se trata de un estudio retrospectivo, descriptivo-analítico, cuantitativo, realizado en un centro de rehabilitación especializado de una ciudad en São Paulo, con una encuesta de 134 registros de pacientes sometidos a Reconstrucción del Tránsito Intestinal (RTI), aprobado por el Comité de Ética. en Investigación (parecer No. 2.831.709). Resultados y discusión: Con el análisis de las historias clínicas de la población de pacientes que se sometieron a RTI 134 (100\%), se obtuvo una edad promedio de 57,3 años, predominantemente masculina 78 (58\%), de la asistencia por parte del sistema público de salud. salud 122 (91\%); diagnóstico oncológico 63 (47\%); cirugía urgente 88 (66\%); y $92(69 \%)$ personas con colostomías. Hubo una correlación estadísticamente significativa entre el tipo de ostomía, el carácter de la cirugía, la técnica quirúrgica y el diagnóstico de indicación quirúrgica, a diferencia de las variables de la cirugía y el tiempo de la ostomía. Conclusión: Existe la necesidad de la prevención y el diagnóstico precoz del cáncer colorrectal, además de planificar la atención de salud para el seguimiento del control quirúrgico y oncológico, para agilizar la RTI cuando sea posible, además de preparar a esta clientela para los cambios. en después de RTI, así como en la gestión de la previsión y provisión de equipos de recolección y adyuvantes, lo que requiere la integración de las acciones de los diferentes niveles de servicio de esta clientela.

Palabras clave: Estomas quirúrgicos; Atención al paciente; Perfil de salud.

\section{Introdução}

As estomias intestinais são confeccionadas em cirurgias para o tratamento de doenças do aparelho digestório (Ambe et al., 2018; Plasencia \& Bahna 2019), sendo que no Brasil um dos principais diagnósticos que tem determinado a sua indicação tem sido o câncer colorretal (CCR), que constitui um importante problema de saúde pública, dentre as condições crônicas não transmissíveis (INCA,2019).

A necessidade da estomia e do uso de equipamentos coletores resultam em mudanças na rotina de vida, alterando a autoimagem e a autoestima, deflagrando preocupações que vão além da doença, que levou a construção da estomia, reconhecida como deficiência física pelo Decreto no. 5296 de 02 de dezembro de 2004 (Brasil, 2004), que requer cuidados especializados, sendo imprescindível o ensino do autocuidado, tanto para o paciente como familiar cuidador (Seo, 2019; Villa et al., 2019). Além disso, a doença que definiu a necessidade a estomização também interfere no processo de aceitação, pois quando envolve diagnóstico oncológico é vinculado à dor, sofrimento e morte, com não aceitação de ter que viver com a estomia. E em geral, as pessoas com doença inflamatória intestinal apresentam um comportamento mais positivo por considerarem que esta condição lhes possibilitará a continuidade da vida (Hueso-Montoro. et al., 2016; Silva et al., 2017). Para o atendimento especializado desta 
população foram estabelecidas as Diretrizes Nacionais para a Atenção à Saúde de Pessoas Ostomizadas no SUS, em 2009 pela Portaria 400 (Brasil, 2009).

As estomias intestinais podem ser provisórias ou definitivas, a depender do tipo de cirurgia, diagnóstico e evolução da doença, assim como da resposta clínica do paciente às terapêuticas instituídas, que podem interferir no prognóstico do paciente (Ambe et al., 2018; Plasencia \& Bahna, 2019). Dentre os aspectos importantes do prognóstico, a possibilidade de realizar a reconstrução de trânsito intestinal (RTI) tem sido valorizado pelos pacientes e tem definido um enfrentamento diferente do adoecimento, suscitando sentimentos de esperança e de finalização do ciclo de sofrimento (Hueso-Montoro. et al., 2016; Silva et al., 2017). No entanto, este procedimento apresenta possíveis riscos de complicações como deiscência, infecção do sítio cirúrgico, assim como outras complicações que podem evoluir para a morte e o ensino do autocuidado podem favorecer a prevenção destas complicações, bem como o alcance da reabilitação (Hardiman et al., 2016; Fonseca et al., 2017; Ambe et al., 2018; Seo, 2019; Sasaki et al., 2020, Sasaki et al., 2021).

Fatores como o envelhecimento e presença de doenças crônicas podem interferir na realização da RTI, o que aumentam as taxas de morbimortalidade, bem como o tempo de permanência com a estomia e a necessidade de uso de equipamentos coletores e de seguimento especializado em nível de atendimento secundário no Sistema Único de Saúde (SUS), que influencia na previsão e provisão de recursos humanos e de equipamentos coletores e adjuvantes de proteção e segurança para esta clientela (Brasil, 2009; Aguiar. et al., 2017; Sasaki et al., 2020). Portanto, o conhecimento das características da população submetida à RTI contribuirá no planejamento e no gerenciamento da assistência especializada à saúde e dos recursos disponíveis no SUS, estabelecida pela Portaria 400.

Este estudo objetivou analisar as características sociodemográficas, clínicas e terapêuticas de pessoas, submetidas à Reconstrução de Trânsito Intestinal.

\section{Metodologia}

Trata-se de um estudo retrospectivo, descritivo-analítico, quantitativo, o qual tem como objetivo descrição das características de usuários cadastrados no Serviço de Atenção ao Estomizado do Centro Especializado em Reabilitação - CER II do interior paulista, referência para 90 municípios da região, composta pelo município sede, além de 30 municípios da microrregião e 59 municípios da macrorregião (Gil, 2010).

A população do estudo constituiu-se dos 134 prontuários de cadastramento das pessoas neste serviço especializado, mediante os critérios de inclusão: ter idade igual ou superior a 18 anos, residentes em um dos municípios da microrregião ou no município sede e ter realizado a reconstrução do trânsito intestinal, no período entre janeiro de 2010 e agosto de 2018; e o critério de exclusão foi prontuário com insuficiência de dados, pertinentes ao estudo.

A coleta de dados ocorreu em setembro de 2018, utilizando-se um instrumento, elaborado pelos pesquisadores, com base na experiência clínica e na revisão de literatura científica, que contemplou informações sociodemográficas (sexo, idade na confecção do estoma) e clínicas (data da cirurgia, diagnóstico de indicação do tratamento cirúrgico com estomização, temporalidade e tipo de estomia, tratamentos realizados, equipamentos coletores/adjuvantes utilizados e presença de complicações de estomia e de pele periestoma).

Respeitando a Resolução CNS 466/12 para pesquisas envolvendo seres humanos, este projeto foi aprovado pelo Comitê de Ética em Pesquisa da Instituição proponente, sob parecer n ${ }^{\circ} 2.831 .709$.

Os dados coletados foram compilados em planilha do Microsoft Excel $® 2013$, com análise descritiva das variáveis no Programa SPSS®versão 20.0., apresentados em tabelas de contingência. Para a análise de correlação aplicou-se o teste do Quiquadrado de Pearson ou o teste de Fischer, conforme pertinência. Adotou-se o nível de significância estatística de 5\%. 


\section{Resultados}

Os dados foram obtidos de 134 prontuários de pessoas que realizaram a RTI, sendo a maioria do sexo masculino 78 (58 \%), média de idade de 57,3 anos, dependentes exclusivamente do SUS (122 - 91\%), com desvio padrão \pm de 14,5; e mediana de 59,5; a idade mínima foi de 21 anos e máxima de 86 anos.

Tabela 1. Variáveis sociodemográficas, clínicas e de tratamento cirúrgico da população do estudo, 2021.

\begin{tabular}{|c|c|c|c|}
\hline Variáveis da clientela & & $\mathrm{N}$ & $\%$ \\
\hline \multirow{2}{*}{ Sexo } & Masculino & 78 & 58 \\
\hline & Feminino & 56 & 42 \\
\hline \multirow{7}{*}{ Faixa etária } & $20-30$ & 4 & 3 \\
\hline & $31-40$ & 13 & 10 \\
\hline & $41-50$ & 25 & 19 \\
\hline & $51-60$ & 31 & 23 \\
\hline & $61-70$ & 40 & 30 \\
\hline & $71-80$ & 14 & 10 \\
\hline & 81 ou mais & 7 & 5 \\
\hline \multirow{2}{*}{ Serviço de Saúde } & Público & 122 & 91 \\
\hline & Privado & 12 & 9 \\
\hline \multirow{6}{*}{ Diagnóstico } & Neoplasias & 63 & 47 \\
\hline & Diverticulite & 28 & 21 \\
\hline & Abdômen agudo & 15 & 11 \\
\hline & Causas Externas & 10 & 7 \\
\hline & Infecções perineais & 8 & 6 \\
\hline & Outras & 10 & 7 \\
\hline \multirow{2}{*}{ Tipo de Estoma } & Colostomia & 92 & 69 \\
\hline & Ileostomia & 42 & 31 \\
\hline \multirow{5}{*}{ Tipo de Cirurgia } & Retossigmoidectomia & 62 & 46 \\
\hline & Colectomia & 38 & 28 \\
\hline & Tratamento paliativo & 15 & 11 \\
\hline & Laparotomia & 16 & 12 \\
\hline & Outras & 3 & 2 \\
\hline \multirow{2}{*}{ Caráter da Cirurgia } & Urgência & 88 & 66 \\
\hline & Eletiva & 46 & 34 \\
\hline
\end{tabular}

Fonte: Dados do pesquisador.

Houve predomínio do diagnóstico oncológico que resultou na confecção do estoma intestinal 63 (47\%), sendo que a cirurgia de Retossigmoidectomia foi a mais frequente em 62 (46\%), confeccionou-se 92 (69\%) colostomias e 88 (66\%) foram cirurgias de urgência.

A indicação de estomização foi correlacionada à idade (Tabela 2), sendo que as pessoas com mais de 41 anos tiveram como causas, as neoplasias e abdômen agudo e àquelas com menos de 40 anos, proporcionalmente em menor número, a estomização foi decorrente de causas externas e abdômen agudo. 
Tabela 2. Caracterização sociodemográfica e de terapêutica cirúrgica da população do estudo, segundo diagnóstico de indicação da estomização, 2021.

\begin{tabular}{|c|c|c|c|c|c|c|c|}
\hline \multirow{3}{*}{ 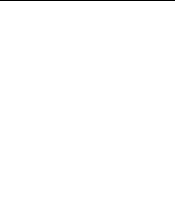 } & \multirow{3}{*}{ Variáveis } & \multicolumn{6}{|c|}{ Diagnóstico de indicação de confecção de estomia } \\
\hline & & $\begin{array}{c}\text { Neoplasias } \\
\text { malignas }\end{array}$ & $\begin{array}{c}\text { Abdome } \\
\text { Agudo }\end{array}$ & Causas Externas & Outras & Total & \multirow[t]{2}{*}{$\mathrm{P}$} \\
\hline & & $\mathrm{N}(\%)$ & $\mathrm{N}(\%)$ & $\mathrm{N}(\%)$ & $\mathrm{N}(\%)$ & $\mathrm{N}(\%)$ & \\
\hline \multirow{7}{*}{ Faixa etária } & $20-30$ anos & ---- & $2(50,0)$ & $1(25,0)$ & $1(25,0)$ & $4(3,0)$ & \multirow{7}{*}{0,001} \\
\hline & $31-40$ anos & $3(23,1)$ & $3(23,1)$ & $5(38,5)$ & $2(15,4)$ & $13(9,7)$ & \\
\hline & $41-50$ anos & $9(36,0)$ & $10(40,0)$ & $1(4,0)$ & $5(20,0)$ & $25(18,7)$ & \\
\hline & $51-60$ anos & $18(58,1)$ & $11(35,5)$ & $1(3,2)$ & $1(3,2)$ & $31(23,1)$ & \\
\hline & $61-70$ anos & $24(60,0)$ & $9(22,5)$ & ---- & $7(17,5)$ & $40(29,9)$ & \\
\hline & $71-80$ anos & $8(57,1)$ & $3(21,4)$ & $1(7,1)$ & $2(14,3)$ & $14(10,4)$ & \\
\hline & 81 anos ou mais & $1(14,3)$ & $5(71,4)$ & $1(14,3)$ & ---- & $7(5,2)$ & \\
\hline \multirow{2}{*}{$\begin{array}{l}\text { Tipo de } \\
\text { estoma }\end{array}$} & Ileostomia & $32(76,2)$ & $5(11,9)$ & $1(2,4)$ & $4(9,5)$ & $42(31,3)$ & \multirow{2}{*}{$\leq 0,000$} \\
\hline & Colostomia & $31(33,7)$ & $38(41,3)$ & $9(9,8)$ & $14(15,2)$ & $92(68,7)$ & \\
\hline \multirow{3}{*}{$\begin{array}{l}\text { Tempo com } \\
\text { estomia }\end{array}$} & Até 1 ano & $32(51,6)$ & $19(30,6)$ & $4(6,5)$ & $7(11,3)$ & $62(46,3)$ & \multirow{3}{*}{0,646} \\
\hline & 1,1 a 2,11 anos & $25(48,1)$ & $17(32,7)$ & $4(7,7)$ & $6(11,5)$ & $52(38,8)$ & \\
\hline & 3 anos ou mais & $6(30,0)$ & $7(35,0)$ & $2(10,0)$ & $5(25,0)$ & $20(14,9)$ & \\
\hline \multirow{4}{*}{ Cirurgia } & Retossigmoidectomia & $37(59,7)$ & $19(30,6)$ & $3(4,8)$ & $3(4,8)$ & $62(46,3)$ & \multirow{4}{*}{0,001} \\
\hline & Colectomia & $23(42,6)$ & $20(37,0)$ & $4(7,4)$ & $7(13,0)$ & $54(40,3)$ & \\
\hline & Confecção de estomia & $3(20,0)$ & $3(20,0)$ & $2(13,3)$ & $7(46,7)$ & $15(11,2)$ & \\
\hline & Outra & ---- & $1(33,3)$ & $1(33,3)$ & $1(33,3)$ & $3(2,2)$ & \\
\hline \multirow{3}{*}{$\begin{array}{l}\text { Caráter } \\
\text { cirúrgico }\end{array}$} & Eletivo & $38(82,6)$ & $2(4,3)$ & $\begin{array}{ll}--- \\
\end{array}$ & $6(13,0)$ & $46(34,3)$ & \multirow{2}{*}{$\leq 0,000$} \\
\hline & Urgência & $25(28,4)$ & $41(46,6)$ & $10(11,4)$ & $12(13,6)$ & $88(65,7)$ & \\
\hline & Total & $63(47,0)$ & $43(32,1)$ & $10(7,5)$ & $18(13,4)$ & $134(100,0)$ & \\
\hline
\end{tabular}

Valor p dos testes de Qui-quadrado, de Pearson ou de Fischer, sendo $\mathrm{P}<0,05$. Fonte: Dados do pesquisador.

As variáveis tipo de estomia, caráter da cirurgia e técnica cirúrgica foram correlacionados aos diagnósticos oncológicos, que predominaram e definiram a necessidade de estomização (Tabela 3). A Retossigmoidectomia e a Colectomia foram as técnicas cirúrgicas mais realizadas, tanto para os casos de neoplasias como de abdômen agudo, em caráter de urgência, sendo que no computo geral, houve maior confecção de colostomias. Ressalta-se que o quantitativo de ileostomias e de colostomias, resultantes por neoplasias malignas foi semelhante.

O diagnóstico de indicação da confecção da estomia não apresentou correlação com a temporalidade desta condição nesta população. 
Tabela 3. Terapêutica cirúrgica da população do estudo, segundo variáveis tempo e tipo de estomização, 2021.

\begin{tabular}{|c|c|c|c|c|c|}
\hline & \multirow[b]{2}{*}{ Variáveis } & \multicolumn{3}{|c|}{ Caráter da Cirurgia } & \multirow[t]{2}{*}{$\mathrm{p}^{*}$} \\
\hline & & Eletivo & Urgência & Total & \\
\hline \multirow{2}{*}{ Tipo de Estomia } & Ileostomia & $30(71,4)$ & $12(28,6)$ & $42(31,3)$ & \multirow{2}{*}{$\leq 0,000$} \\
\hline & Colostomia & $16(17,4)$ & $76(82,6)$ & $92(68,7)$ & \\
\hline \multirow{3}{*}{ Tempo de estomia } & Até 1 ano & $21(33,9)$ & $41(66,1)$ & $62(46,3)$ & \multirow{3}{*}{0,994} \\
\hline & De 1,1 a 2,11 anos & $18(34,6)$ & $34(65,4)$ & $52(38,8)$ & \\
\hline & 3 anos ou mais & $7(35,0)$ & $13(65,0)$ & $20(14,9)$ & \\
\hline \multirow{4}{*}{ Cirurgia } & Retossigmoidectomia & $31(50,0)$ & $31(50,0)$ & $62(46,3)$ & \multirow{4}{*}{0,002} \\
\hline & Colectomia & $13(24,1)$ & $41(75,9)$ & $54(40,3)$ & \\
\hline & Confecção de estoma & $1(6,7)$ & $14(93,3)$ & $15(11,2)$ & \\
\hline & Outras & $1(33,3)$ & $2(66,7)$ & $3(2,2)$ & \\
\hline \multicolumn{2}{|r|}{ Total } & $46(34,3)$ & $88(65,7)$ & $134(100,0)$ & \\
\hline
\end{tabular}

*Valor p dos testes de Qui-quadrado, de Pearson ou de Fischer, sendo $\mathrm{P}<0,05$. Fonte: Dados do pesquisador.

O caráter da cirurgia foi correlacionado à técnica cirúrgica realizada e ao tipo de estomia confeccionado, sendo majoritariamente às de urgência (Tabela 4). As técnicas de Retossigmoidectomia e Colectomia foram as mais empregadas, nas cirurgias de urgência. As colostomias prevaleceram nas cirurgias de urgência. Contudo, não houve correlação positiva entre a cirurgia realizada e o tempo de permanência da estomia nesta população.

Tabela 4. Tempo de estomização, segundo variáveis faixa etária e tipo de estomia. Ribeirão Preto, Brasil, 2021

\begin{tabular}{|c|c|c|c|c|c|c|}
\hline \multirow{2}{*}{\multicolumn{2}{|c|}{ Variáveis }} & \multicolumn{5}{|c|}{ Temporalidade da estomia intestinal } \\
\hline & & \multirow{2}{*}{$\begin{array}{l}\text { Até } 1 \text { ano } \\
4(100,0)\end{array}$} & \multirow{2}{*}{$\frac{1,1 \text { a } 2,11 \text { anos }}{----}$} & \multirow{2}{*}{$\begin{array}{c}\begin{array}{c}3 \text { anos } \\
\text { ou mais }\end{array} \\
----\end{array}$} & \multirow{2}{*}{$\begin{array}{l}\text { Total } \\
4(3,0)\end{array}$} & \multirow{2}{*}{$\mathrm{p}^{*}$} \\
\hline \multirow{7}{*}{ Faixa etária } & 20 a 30 anos & & & & & \\
\hline & 31 a 40 anos & $4(30,8)$ & $8(61,5)$ & $1(7,7)$ & $13(9,7)$ & \multirow{6}{*}{0,084} \\
\hline & 41 a 50 anos & $11(44,0)$ & $7(28,0)$ & $7(28,0)$ & $25(18,7)$ & \\
\hline & 51 a 60 anos & $18(58,1)$ & $11(35,5)$ & $2(6,5)$ & $31(23,1)$ & \\
\hline & 61 a 70 anos & $16(40,0)$ & $20(50,0)$ & $4(10,0)$ & $40(29,9)$ & \\
\hline & 71 a 80 anos & $6(42,9)$ & $4(28,6)$ & $4(28,6)$ & $14(10,4)$ & \\
\hline & 81 anos ou mais & $3(42,9)$ & $2(28,6)$ & $2(28,6)$ & $7(5,2)$ & \\
\hline \multirow{2}{*}{ Tipo de estomia } & Ileostomia & $22(52,4)$ & $18(42,9)$ & $2(4,8)$ & $42(31,3)$ & \multirow{2}{*}{0,083} \\
\hline & Colostomia & $40(43,5)$ & $34(37,0)$ & $18(19,6)$ & $92(68,7)$ & \\
\hline \multicolumn{2}{|c|}{ Total } & $62(46,3)$ & $52(38,8)$ & $20(14,9)$ & $134(100,0)$ & \\
\hline
\end{tabular}

*Valor p dos testes de Qui-quadrado, de Pearson ou de Fischer, sendo $\mathrm{P}<0,05$. Fonte: Dados do pesquisador.

Não houve correlação estatisticamente significante entre a idade, o tipo e a temporalidade da estomia intestinal, sendo que a maioria da população permaneceu com a estomia por um período de até 2,11 anos.

\section{Discussão}

A média de idade dos pacientes, que realizaram a RTI foi de 57,3 anos, com predomínio do sexo masculino, de neoplasia maligna, de colostomia e de cirurgia de urgência, corroborando com o perfil epidemiológico brasileiro, que tem indicado o 
aumento da prevalência das doenças crônicas não transmissíveis (DCNT), destacando-se o de câncer de colorretal (CCR), que tem constituído uma das principais causas de confecção de estomia intestinal. O desenvolvimento do CCR está associado à fatores de risco não modificáveis e modificáveis, sendo que estes últimos possibilitam a abordagem preventiva dos hábitos de vida, por meio da alimentação saudável, combate ao sedentarismo e controle da obesidade, juntamente com o rastreamento de CCR na Atenção Primária Saúde, para diminuir, ou ao menos, possibilitar o diagnóstico e tratamento precoce de CCR (INCA, 2019).

A realização das cirurgias Retossigmoidectomia e a Colectomia indicou a ocorrência de CCR em estadiamento mais avançado, com ressecção da porção final do colón e do reto, tanto em caráter de urgência como eletivo (Plasencia \& Bahna, 2019), que requereu maior densidade tecnológica e equipes de saúde especializadas, assim como de instituições terciárias. A necessidade da realização da cirurgia de urgência evidenciou o CCR em estadiamento avançado, sendo que esta situação pode ser resultar em obstrução parcial ou total da luz intestinal, que também pode ser diagnosticado como abdômen agudo, possivelmente incluídos nos 43 (32,1\%) casos (Bayar et al. 2015; Ambe et al., 2018; Thompson et al., 2019). A cirurgia de urgência impossibilita o preparo físico e psicoemocional adequado do paciente, o que pode gerar maior estresse e fadiga fisiológica no pós-operatório, agravando ainda mais a fadiga oncológica, aplicável para 63 (47,0\%) da população deste estudo.

Embora o emprego das técnicas de Retossigmoidectomia e Hartmanm para o tratamento destes diagnósticos, que acometiam o colón e o reto, seja um consenso mundial estabelecido entre os cirurgiões, que prevê a confecção de estomia, estudos têm associado estes procedimentos às maiores taxas de morbimortalidade e às menores taxas de RTI (Horesh et al., 2017; Qureshi et al., 2018; Plasencia \& Bahna, 2019). Consequentemente, isto pode determinar maior tempo de estomização devido ao comprometimento clínico e ocorrência de complicações pós-operatórias, que impactará na qualidade de vida, necessidade de seguimento multiprofissional especializado por maior tempo e o uso de equipamentos coletores e adjuvantes, aumentando o ônus ao SUS (Horesh et al., 2017; Qureshi et al., 2018; Thompson et al., 2019; Sasaki et al., 2020).

Enfatiza-se que o custo mensal é maior com os equipamentos coletores e adjuvantes para as pessoas com colostomias, que predominou neste estudo, onerando a manutenção dos serviços de assistência especializada (Lira et al., 2019) e reforça a necessidade de rastreamento e diagnóstico precoce de CCR, para viabilizar o planejamento cirúrgico eletivo, sendo que em muitos casos, não será necessária a estomização. Consequentemente, a ação preventiva na Atenção Primária à Saúde poderá reduzir a necessidade de cirurgias de urgência com construção de colostomias, além de dirimir a ocorrência de complicações como infecção do sítio cirúrgico e maior comprometimento clínico relacionadas aos procedimentos de urgência. Por outro lado, nos casos com estomização, favoreceria a RTI, em menor tempo de pós-operatório, contribuindo para a resolutividade da Rede de Assistência à Saúde no SUS (Bayar et al. 2015; Thompson et al., 2019; Plasencia \& Bahna, 2019).

Por outro lado, o tempo de permanência com estomia não apresentou nível de significância estatística em relação à causa da estomia, caráter da cirurgia e faixa etária, possivelmente pelo fato de o tratamento oncológico depender de outras variáveis como evolução e resposta clínica do paciente à cirurgia, assim como necessidade de tratamentos adjuvantes como quimioterapia antineoplásica e radioterapia, inclusive com associação destas, a quimiorradioterapia (Ambe et al., 2018). Ainda, independente do diagnóstico, outros fatores como complicações pós-cirúrgicas e presença de comorbidades relacionadas ao envelhecimento, podem refletir na indicação da RTI, postergando a sua realização, o que pode ser ratificado pelo tempo de estomização acima de 1,1 anos, chegando a ser superior a três anos. Em geral, na ausência de complicações ou de maior comprometimento clínico do paciente, a indicação de RTI é realizada com três meses (Aguiar et al., 2018).

Houve predomínio do diagnóstico oncológico, que resultou majoritariamente na confecção de colostomia, cuja localização tumoral na porção do cólon e reto, possibilitou a sua ressecção parcial. Além disso, se houver comprometimento total do cólon e reto por câncer, será realizada a ressecção total com confecção de ileostomia. Portanto, a depender da localização do tumor ou da lesão por outras causas não oncológicas, a ressecção da alça intestinal pode resultar em diferentes tipos de 
estomias, com resíduo fecal de diferentes características, que determinarão cuidados específicos e a indicação de equipamentos coletores e adjuvantes com características, que possam proporcionem segurança e proteção à pele periestoma (Seo, 2019).

Quando ocorre a indicação de cirurgia de urgência, focaliza-se a resolução do problema e das queixas do paciente e por envolver risco de morte, pode inviabilizar a realização de exames especializados, que por conseguinte pode comprometer a resolutividade do tratamento e a recuperação do paciente (Ambe et al., 2018; Thompson et al., 2019).

O fato da estomização ter sido correlacionada à faixa etária acima de 41 anos, indica o perfil epidemiológico e a representatividade do CCR como uma das condições crônicas importantes para a população acima desta faixa etária, o que deve ser considerado no planejamento da assistência à saúde no SUS, com intervenções de promoção e prevenção de adoecimento crônico (INCA, 2019).

A indicação da RTI depende da evolução e da resposta clínica do paciente ao tratamento cirúrgico e pelo predomínio da neoplasia nesta população, que requer seguimento de controle oncológico de cinco anos, a demora para a sua realização pode ser justificada pela necessidade de tratamentos adjuvantes ou evolução com piora clínica importante, que inviabiliza este procedimento (Aguiar et al., 2017; Bayar et al., 2016; Fonseca et al., 2017; Horesh et al., 2017; Qureshi et al., 2018). Por outro lado, outros diagnósticos também indicaram a gravidade clínica dos pacientes para a realização da cirurgia de urgência, o que também suscita a necessidade de revisão da implementação das ações preventivas e de promoção de saúde na Atenção Primária à Saúde.

No contexto hospitalar, a assistência especializada implica em intervenções de enfermagem com ensino pré-operatório aliada à demarcação de estoma, ensino de autocuidado com estoma e equipamentos coletores/adjuvantes para paciente e familiar cuidador, bem como a alta responsável para os serviços secundários de atendimento aos estomizados (Hardiman et al., 2016; Seo, 2019; Villa et al., 2019; Sasaki et al., 2020). E a assistência a estes pacientes devem incluir a avaliação periódica em relação à condição geral, à evolução clínica e a respostas aos tratamentos, assim como em relação à utilização e indicação destes equipamentos coletores e a possibilidade de RTI, além dos aspectos psicossociais (Hueso-Montoro et al., 2016; Silva et al., 2017; Aguiar et al., 2018; Lira et al, 2019; Sasaki et al., 2020; Sasaki et al., 2021).

A estomia impacta na qualidade de vida, na autoimagem e na autoestima da pessoa, que necessita utilizar equipamentos/adjuvantes, que têm custo elevado e, portanto, o seguimento especializado pelas equipes multiprofissionais deve focalizar o autocuidado (Hardiman et al., 2016; Silva et al, 2017; Seo, 2019; Villa et al., 2019; Sasaki et al., 2020; Sasaki et al., 2021) estar articulado e integrado à Rede de Atenção à Saúde, para a redução de complicações e do tempo para a RTI, bem como preparo destas pessoas para as mudanças pós RTI, considerando-se o preparo e reabilitação pélvica (Hardiman et al., 2016; Qureshi et al., 2018).

Os resultados deste estudo indicam a necessidade do estabelecimento de uma Linha de cuidado para esta população, para a determinação de um fluxograma de assistência à saúde, desde a entrada na Atenção Primária à Saúde, tratamento e seguimento ambulatorial especializado. Portanto, deve-se organizar a oferta de ações de promoção, prevenção, tratamento e reabilitação em saúde no SUS, por uma equipe multidisciplinar para assegurar a continuidade da assistência (Franco, Magalhães \& Júnior, 2011). Para tanto, deve-se centrar nas necessidades da população para estabelecer projeto terapêutico adequado, uso de tecnologias leve, assim como uma rede de serviços, com recursos assistenciais disponíveis, levando-se em conta os determinantes sociais e a regulação deste sistema (Malta \& Merhy, 2010). Isso pressupõe a capacitação dos profissionais da equipe multiprofissional de saúde, para assegurar a efetividade da linha de cuidado.

As limitações desta pesquisa estão relacionadas ao fato de ter constituído um estudo transversal, que abordou os pacientes submetidos à RTI em um único serviço de assistência especializada para estomizados, assim como não houve a identificação dentre os cadastrados, daqueles que, apesar de apresentarem condições clínicas para RTI, não haviam tido a acessibilidade ao procedimento. 


\section{Conclusão}

A população submetida à RTI apresentou média de idade de 57,3 anos, sendo 58\% do sexo masculino, $91 \%$ dependente do SUS, $47 \%$ com diagnóstico oncológico, 69\% com colostomia e 66\% foram submetidos à cirurgia de urgência. Houve correlação positiva entre tipo de estomia, caráter da cirurgia, técnica cirúrgica e o diagnóstico de indicação cirúrgica; mas não houve correlação estatisticamente significante entre cirurgia e tempo de estomia.

Estes resultados apontaram a necessidade de estratégias para a prevenção e o diagnóstico precoce do CCR na Rede de Atenção à Saúde no SUS para viabilizar tratamento precoce com cirurgias eletivas e, portanto, maior viabilidade de RTI, com menor tempo de pós-operatório.

A assistência especializada deve ocorrer nos diferentes níveis de atenção à saúde no SUS, com melhor integração entre si, considerando-se as características sociodemográficas, clínicas e terapêuticas da população assistida, para a obtenção de maior efetividade assistencial, melhor gestão dos recursos humanos, materiais e de infraestrutura para otimização dos recursos públicos, que pode ser alcançado com o estabelecimento da Linha de cuidado para esta população.

Espera-se que os resultados deste estudo possam subsidiar outros estudos sobre a necessidade de avaliação de cadastrados que possam ser beneficiados com a RTI com menor tempo de estomização.

\section{Referências}

Aguiar, J. C. et al. (2017). Clinical and sociodemographic aspects of people with a temporary intestinal stoma. Revista Mineira de Enfermagem. 21(1), 1-7. http://www.dx.doi.org/10.5935/1415-2762.20170023

Aguiar, J. C., Pereira, A. P. S., \& Pinto, M. H. (2018). Reconstrução de trânsito intestinal: fatores que influenciam a realização. Rev. Eletr. Enf. 20 (1), 20-32. https://doi.org/10.5216/ree.v20.47606.

Ambe, P. C., Kurz, N. R., Nitschke, C., Odeh, S. F., Möslein, G., \& Zirngibl, H. (2018). Intestinal ostomy-classification, indications, ostomy care and complication management. Dtsch Arztebl Int. https://doi.org/10.3238/arztebl.2018.0182

Bayar, B., Yilmaz, K. B., Akinci, M., Sahin, A., \& Kulacoglu, H. (2016). An evaluation of treatment results of emergency versus elective surgery in colorectal cancer patients. Turkish Journal of Surgery. 32 (1), 11-17. https://dx.doi.org/10.5152\%2FUCD.2015.2969

Brasil. Ministério da Saúde. (2004). Decreto $\mathrm{n}^{\mathrm{o}} 5.296$ de 2 de Dezembro de 2004. <http://www.planalto.gov.br/ccivil_03/_ato2 0042006/2004/decreto/d5296.htm>.

Brasil. Ministério da Saúde. Secretaria de Atenção à Saúde. (2009). Portaria n n $^{\circ} 400$, de $16 \quad$ de novembro de 2009 . <http://bvsms.saude.gov.br/bvs/saudelegis/sas/2009/prt0400_16_11_2009.html>.

Fonseca, A. Z., Uramoto, E., Santos-Rosa, O. M., Santin, S., \& Ribeiro-Jr, M. (2017). Colostomy closure: risk factors for complications. ABCD, arq. bras. cir. Dig. 30 (4), 231-234. https://doi.org/10.1590/0102-6720201700040001

Franco, T. B., \& Magalhães Júnior, H, M. (2011). Integralidade na assistência à saúde: organização das linhas de cuidado. In: Merhy EE, Magalhães Júnior HM, Rimoli J, Franco TB, Bueno WS, organizadores. O trabalho em saúde: olhando e experienciando o SUS no cotidiano. (3a ed.), Hucitec.

Gil, A. C. (2010). Como elaborar projetos de pesquisa. (5a ed.), Atlas.

Hardiman, K. M., Reames, C. D., Mcleod, M. C., \& Regenbogen, S. E. (2016). Patient autonomy-centered self-care checklist reduces hospital readmissions after ileostomy creation. Surgery. 160(5), 1302-1308. https://doi.org/10.1016/j.surg.2016.05.007

Horesh, N., Lessing, Y., Rudnicki, Y., Kent, I., Kammar, H, Ben-Yaacov, A, et.al. (2017). Considerations for Hartmann's reversal and Hartmann's reversal outcomes - a multicenter study. International Journal of Colorectal Disease. 32(11), 1577-1582. https://doi.org/10.1007/s00384-017-2897-2

Hueso-Montoro, C. et al. (2016). Experiences and coping with the altered body image in digestive stoma patients. Revista Latino-americana de Enfermagem, 24(1), 1-9. https://doi.org/10.1590/1518-8345.1276.2840

Instituto Nacional de Câncer José Alencar Gomes da Silva (INCA). (2019). Estimativa 2020: incidência de câncer no Brasil. https://www.inca.gov.br/sites/ufu.sti.inca.local/files//media/document//estimativa-2020-incidencia-de-cancer-no-brasil.pdf

Lira, J. A. C. et al. (2019). Custos de equipamentos coletores e adjuvantes em pacientes com estomias de eliminação. Rev Min Enferm. http://www.dx.doi.org/10.5935/1415-2762.20190011

Malta, D. C., \& Merhy, E. E. (2010). O percurso da linha do cuidado sob a perspectiva das doenças crônicas não transmissíveis. Interface - Comunicação, Saúde, Educação, 14(34), 593-606. https://doi.org/10.1590/S1414-32832010005000010

Plasencia, A., \& Bahna, H. (2019). Diverting Ostomy: For Whom, When, What, Where, and Why. Clin Colon Rectal Surg. 32(3), 171-175. https://pubmed.ncbi.nlm.nih.gov/31061646/ 
Research, Society and Development, v. 10, n. 6, e4410615241, 2021

(CC BY 4.0) | ISSN 2525-3409 | DOI: http://dx.doi.org/10.33448/rsd-v10i6.15241

Qureshi, A., Cunningham, J., \& Hermandas, A. (2018). Elective vs. Emergency Stoma Surgery Outcomes. World J Surg Surgical Res. http://www.surgeryresearchjournal.com/full-text/wjssr-v1-id1050.php

Sasaki, V. D. M, Teles, A. A. S, Russo, T. M. S, Aguiar, J. C, Paraizo-Horvath, C. M. S, \& Sonobe, H. M. (2020). Care in the Ostomates Programs: the multidisciplinary team's perspective. Rev Rene. https://doi.org/10.15253/2175-6783.20202144295

Sasaki, V. D. M., Teles, A. A. S, Silva, N. M., Russo, T. M. S., Pantoni, L. A., Aguiar, J. C., et al. (2021). Self-care of people with intestinal ostomy: beyond the procedural towards rehabilitation. Rev Bras Enferm.74(1). http://dx.doi.org/10.1590/0034-7167-2020-0088

Seo, H-W. (2019). Effects of the frequency of ostomy management reinforcement education on self-care knowledge, self-efficacy, and ability of stoma appliance change among Korean hospitalised ostomates. Int Wound J. 16(1),21-28. https://doi.org/10.1111/iwj.13047

Silva, N. M., Santos, M. A., Rosado, S. R., Galvão, C. M., \& Sonobe, H. M. (2017). Psychological aspects of patients with intestinal stoma: integrative review. Rev. Latino-Am. Enfermagem. https://doi.org/10.1590/1518-8345.2231.2950

Thompson, H. M. et al. (2019). Emergent Versus Elective Ostomies for Colon Cancer: is there a difference? Gastroenterology. https://doi.org/10.1016/S00165085(19)40825-1

Villa, G., Mannarini, M., Giovanna, G. D., Marzo, E., Manara, D. F., \& Vellone, E. (2019). A literature review about self-care on ostomy patients and their caregivers. Int J Urol Nurs. https://doi.org/10.1111/ijun.12182 